\title{
Immediate reattachment of fractured tooth segment using dual cure resin
}

\author{
Joshi $\mathbf{N}^{1}$, Shetty $\mathbf{N}^{2}$, Kundabala $\mathbf{M}^{3}$ \\ ${ }^{1}$ Specialist Resident, ${ }^{2}$ Associate Professor, ${ }^{3}$ Professor and Head, Manipal College of Dental science, Mangalore, India
}

\begin{abstract}
Crown fracture of maxillary anterior teeth is frequent dental injuries for which patients seek immediate dental treatment. Immediate reattachment of the intact fractured tooth segment is a therapeutic choice in such cases. This case report presents one of the best methods used for reattachment, which is an ultraconservative technique. It allows the restoration of original tooth contour, colour and aesthetics.
\end{abstract}

Key words: Crown Fracture, Aesthetics, Dual cure resins

$$
\mathrm{T}_{\mathrm{se}}^{\mathrm{he}}
$$
he maxillary central incisors are the teeth most susceptible to fractures caused by direct trauma such as contact sports, road accidents and falls. Aesthetic rehabilitation of crown fractures of the maxillary anterior is one of the greatest challenges to the dentist. The present generation is very conscious about their appearance and demand for immediate treatment and aesthetic rehabilitation. The conventional approach for rehabilitation of fractured anterior teeth include composite restoration, post supported prosthetic restoration and in some cases extraction and fixed prosthetics ${ }^{1}$. With the introduction of technologically improved composite resins especially dual cured resins and dentin bonding agents, innovative techniques to treat fractured teeth has evolved.

Chosack and Eildeman published the first case report on reattachment of a fractured incisor fragment in $1964^{2}$. Eighty percent of traumatized incisors fracture in an oblique fashion from labial to lingual aspect with fracture line proceeding in a oblique direction ${ }^{2}$. Factors that influence the success of reattachment include the site of fracture, size of fractured remnants, periodontal status, pulpal involvement, maturity of the root formation, biological width invasion, occlusion, time material used for reattachment, use of post, and prognosis ${ }^{3}$. Reattachment of intact natural tooth fragment is advantageous compared to crowns since colour, morphology, transluceny can be retained and treatment is immediate.

\section{Case report}

A 23years old male patient injured during a road accident had sustained a complicated crown root fracture that extended subgingivally in the palatal aspect. He reported to the Department of Conservative dentistry and endodontics, M.C.O.D.S Mangalore immediately after the injury.

On clinical examination tooth was tender on percussion with a mobile coronal fragment. There was bleeding on probing the fragment and no soft tissue injury observed. Radiographic observation revealed a fracture line at the cervical line of the upper right central incisor. On periodontal examination the coronal tooth fragment was still attached palatally by fragile soft tissue.

A treatment plan was formulated to immediately reattach the natural tooth fragment since the patient was concerned about aesthetics. Local anaesthesia was administrated and fractured coronal fragment was removed with a forceps without damage. Pulp chamber was cleaned by removing all pulp tissue and stored in saline. Under rubber dam vital pulp tissue was extirpated from the root canal. Working length determined using Ingles technique and confirmed by apex locator (RootZX).Biomechanical preparation was done by crown down technique, followed by obturation of the canal using lateral condensation technique.

Electrocautary was done to expose the palatal margins. $7 \mathrm{~mm}$ of gutta percha was removed from the canal and a

\footnotetext{
Correspondence

Dr. Neera Joshi

Specialist Resident

Department of Conservative Dentistry and Endodontics

Manipal College of Dental Science, Mangalore

Karnataka, India

E-mail: neera_ktm@yahoo.com
} 
prefabricated parallel serrated post (produits Dentaires SA) was selected. Post was cemented using Panavia F 2.0 (Kuraray Medical Inc). A retention box using no 4 round bur (Diatech) was prepared in the coronal fragment to accommodate the head of the post. The fractured crown segment and the tooth were etched with K Etchant gel for 10 second's. ED Primer was applied on the surface of the tooth structure (enamel

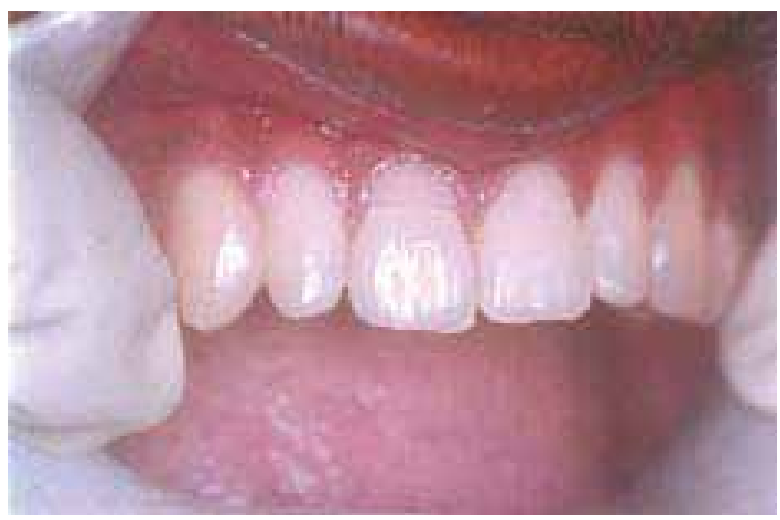

Fig 1: Coronal tooth fracture at the cervical third of the tooth

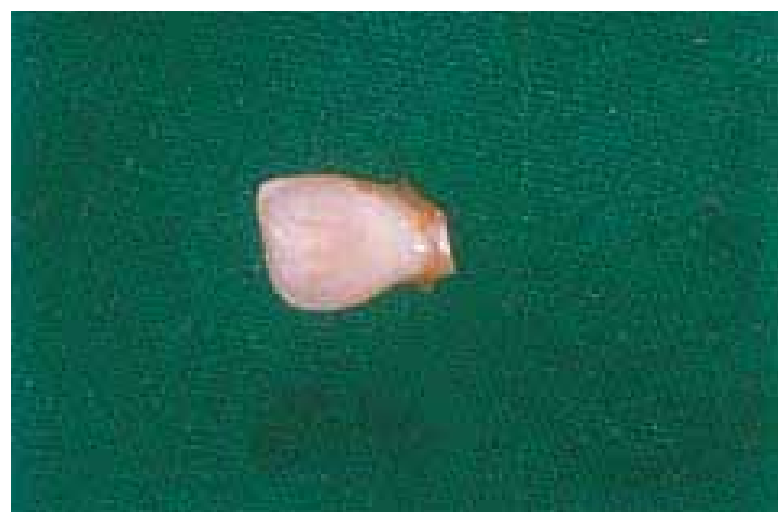

Fig 3: Fractured coronal segment (palatal view)

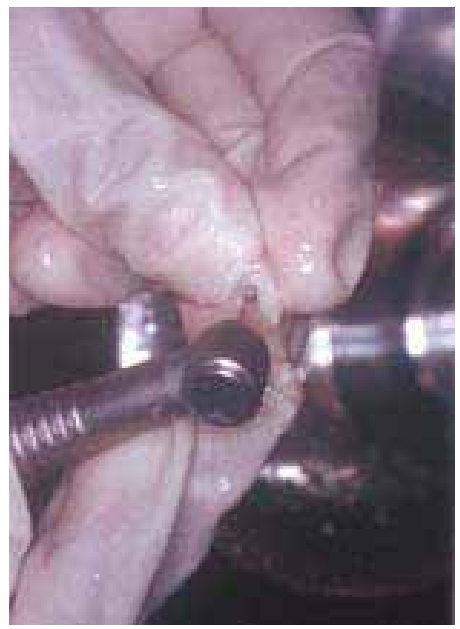

Fig 5: Prepararion of a retention box and dentin) with a disposable brush and left in place for 30 seconds. Panavia F 2.0 paste was mixed and applied around the post and the retention box of the fragment and the fragment was reattached to the tooth. Excess paste along the margins was removed using No 12 BP blade and light cured with Smartlite LED (Dentsply) curing light for 20 seconds. Excess cement adhered to the tooth surface was removed by polishing.

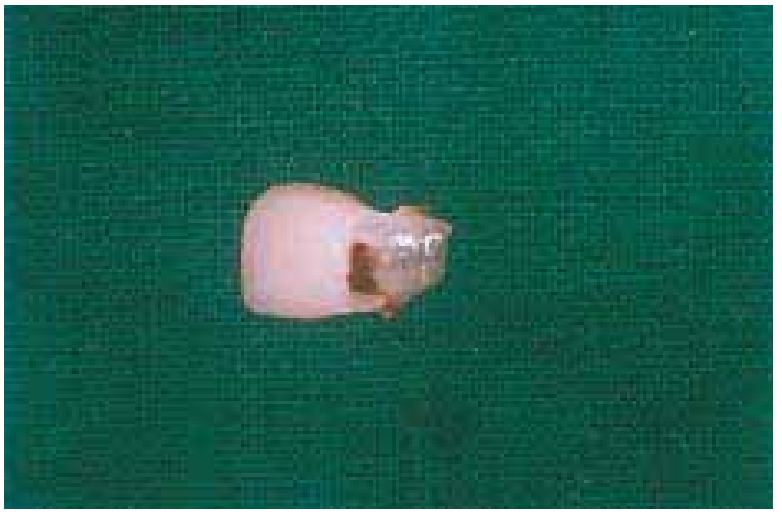

Fig 2: Fractured coronal segment (facial view)

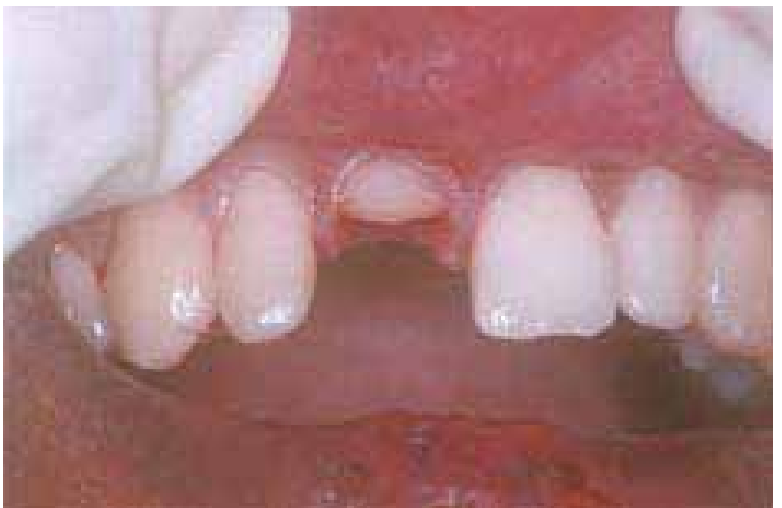

Fig 4: Tooth after removal of fractured segment

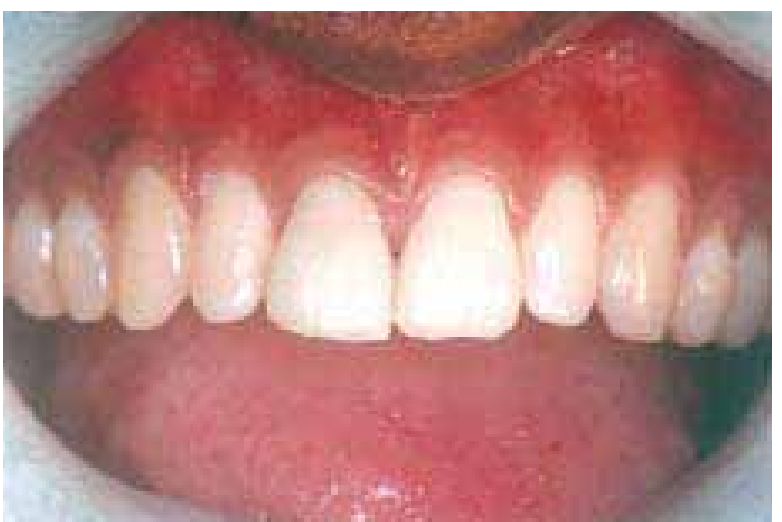

Fig 6: Post operative 


\section{Discussion}

Reattachment of intact coronal fragment is an economical and less time consuming procedure. The reattached tooth is restored to its original form, contour and margins and tends to be more compatible with the gingiva ${ }^{4}$. The psychological trauma caused to the individual due loss of aesthetics can be managed by this procedure successfully. When a tooth has not sustained a luxation injury, this technique should be considered ${ }^{5}$. The wide range of materials available in the market today makes the choice of material difficult. Various materials such as flowable composite, dual cure or resin modified glass ionomers can be used ${ }^{2}$. In this case Panavia F2.0 was used. It is a dual curing (chemical and light cure) resin. The system consist of ED primer 11, Panavia F 2.0 paste and oxyguard 11. The advantage of this system are bond strength, aesthetics, complete curing. The medium of dental fragment conservation is important to maintain fragment hydration. The best medium for a short duration is physiological solution ${ }^{6}$. A prefabricated post was used as it is economical and increases the retention and distributes the forces along the root $^{6}$.

\section{Conclusion}

Reattachment of the intact fractured segment can be considered as an ultraconservative method for aesthetic rehabilitation.This procedure helps us to preserve maximal natural tooth structure. The superior quality adhesive materials make this procedure viable. The need of the day is to educate the population to preserve the fractured segment and seek immediate dental treatment.

\section{References}

1. Attila IO, Cenk MHA, Serdar MT. Multidisciplinary approach to the rehabilitation of a crown -root fracture for immediate esthetics. Dent traumatol. 2006;22(1):48-52.

2. Reis A, Loguercio AD, Kraul A, Matson E. Reattachment of fractured teeth: A review of literature regarding techniques and materials. Oper Dent. 2004;29(2):226-33.

3. Wadhwani CPK. A single visit, multidisciplinary approach to the management of traumatic tooth crown fracture.Br Dent J. 2000;188:593-8.

4. Shivanna V, Dhanya NMK, Babu SS. Management of complicated Crown -Root Fracture in one visit by Re-bonding procedure-A case Report. Karnataka State Dent J. 2006;25:58-9.

5. Giorgio R, Claudia M, Angelo P. Clinical procedures for the immediate reattachment of a tooth fragment. Dent Traumatol. 2002;18:281-4.

6. Pasini S, Bardellini E, Keller E, Conti G, Flocchini P, Majorana A. Surgical removal and immediate reattachment of coronal fragment embedded in lip. Dent Traumatol. 2006;22:165-8. 